\title{
Practical Stabilization of Uncertain Nonholonomic Mobile Robots Based on Visual Servoing Model with Uncalibrated Camera Parameters
}

\author{
Hua Chen, ${ }^{1}$ Bingyan Chen, ${ }^{1}$ Baojun $\mathrm{Li}^{1}{ }^{1}$ and Jinbo Zhang ${ }^{2}$ \\ ${ }^{1}$ Mathematics and Physics Department, Hohai University, Changzhou Campus, Jiangsu, Changzhou 213022, China \\ ${ }^{2}$ Jiangsu Key Laboratory of Power Transmission and Distribution Equipment Technology, Hohai University, Changzhou Campus, \\ Jiangsu, Changzhou 213022, China \\ Correspondence should be addressed to Hua Chen; chenhual12@163.com
}

Received 15 January 2013; Revised 14 April 2013; Accepted 18 April 2013

Academic Editor: Baocang Ding

Copyright ( 92013 Hua Chen et al. This is an open access article distributed under the Creative Commons Attribution License, which permits unrestricted use, distribution, and reproduction in any medium, provided the original work is properly cited.

\begin{abstract}
The practical stabilization problem is addressed for a class of uncertain nonholonomic mobile robots with uncalibrated visual parameters. Based on the visual servoing kinematic model, a new switching controller is presented in the presence of parametric uncertainties associated with the camera system. In comparison with existing methods, the new design method is directly used to control the original system without any state or input transformation, which is effective to avoid singularity. Under the proposed control law, it is rigorously proved that all the states of closed-loop system can be stabilized to a prescribed arbitrarily small neighborhood of the zero equilibrium point. Furthermore, this switching control technique can be applied to solve the practical stabilization problem of a kind of mobile robots with uncertain parameters (and angle measurement disturbance) which appeared in some literatures such as Morin et al. (1998), Hespanha et al. (1999), Jiang (2000), and Hong et al. (2005). Finally, the simulation results show the effectiveness of the proposed controller design approach.
\end{abstract}

\section{Introduction}

For the last few decades, the problem of control of nonholonomic systems has been studied by many researchers and has attracted a continuously increasing attention in the control community. As pointed out in [1], such a class of nonlinear systems cannot be stabilized to a point with pure smooth (or even continuous) state-feedback control. Therefore, the stabilization problem of nonholonomic systems is still extremely challenging. Up to now, there have been a lot of control methods to stabilize such systems: continuous time-varying feedback control laws [2-4], hybrid feedback control laws [57], and discontinuous feedback control laws [8-11].

Recently, based on the visual servoing feedback model, a new robust control issue is considered in [12] for uncertain nonholonomic mobile robots with uncalibrated camera parameters. In [13], an adaptive sliding mode controller is designed to stabilize a class of uncertain dynamic mobile robots in the presence of parametric uncertainties of the camera system. In addition, the dynamic feedback robust regulation problem and the dynamic feedback tracking control problem have been addressed with unknown depth information and visual parameters of a fixed camera in $[14,15]$, respectively. However, these results have a common defect: it may be somewhat unrealistic to assume that some parameters of the camera system are unknown while others are known. And these above existing approaches [2-15] are invalid when we deal with the robust stabilization problem for such nonholonomic mobile robots with assuming that all the camera parameters are unknown.

In addition, as shown in $[16,17]$, the unicycle robot model has dynamic linearizable feedback, based on which some control problems for nonholonomic system have been solved in the recent research literatures. For example, in [18], the unicycle model can be transformed into two double integrators by using dynamic feedback linearization; then a new class of distributed nonlinear controllers for leader-following formation control of unicycle robots without global position measurements has been proposed. But there exists singularity of the transformed model caused by the nonholonomic 
constraint of the mobile robot; how to avoid appearance of singularity is the major difficulty of designing the control law. And in [19], the problem of stabilization for nonholonomic single chained systems has also been considered in the framework of nonregular feedback linearization.

This paper considers the practical stabilization problem for a class of nonholonomic mobile robots based on visual servoing feedback with uncalibrated visual parameters. A new switching controller is presented in the presence of parametric uncertainties. Our main contributions can be summarized in the following three respects.

(1) The new design method is directly based on the original system model without using any transformation of coordinates or inputs; it is valid to avoid appearance of singularity. Under the proposed switching controller, it is rigorously proved that all the states of closed-loop system can be stabilized to a prescribed arbitrarily small neighborhood of the zero equilibrium point.

(2) Compared with other results on the same subject (visual servoing feedback control of nonholonomic mobile robots) [12-15], it is more realistic to suppose that all the parameters of the camera system are unknown.

(3) The switching controller design method can be easily used to solve the practical stabilization problem of a kind of nonholonomic mobile robots with uncertain parameters and angle measurement disturbance raised by [20-23] and references therein.

The structure of this paper is as follows: Section 2 gives a statement of some motivating examples and the problem considered in this paper. A proper assumption and a lemma are also presented in this section. Section 3 gives our main results including switching controller design, stability analysis (proof), and an application. Section 4 provides an illustrative numerical example and the corresponding simulation results of the proposed methodology. Finally, a conclusion is shown in Section 5.

\section{Motivating Examples and Problem Statement}

2.1. Motivating Examples. A class of nonholonomic wheeled mobile robots is shown in Figure 1. The two fixed rear wheels of the robot are controlled independently by motors, and a front castor wheel prevents the robot from tipping over as it moves on a plane. Assume that the geometric center point and the mass center point of the robot are the same, the radii $r$ are identical for all the wheels, and the distance $2 R$ between the fixed wheels is a known positive constant. Its kinematic model can be described by the following differential equations [24]:

$$
\begin{gathered}
\dot{x}=v \cos \theta, \\
\dot{y}=v \sin \theta, \\
\dot{\theta}=\omega,
\end{gathered}
$$

where $(x, y)$ is the position of the mass center of the robot moving on the plane. $v$ is the forward velocity, $\omega$ is the steering

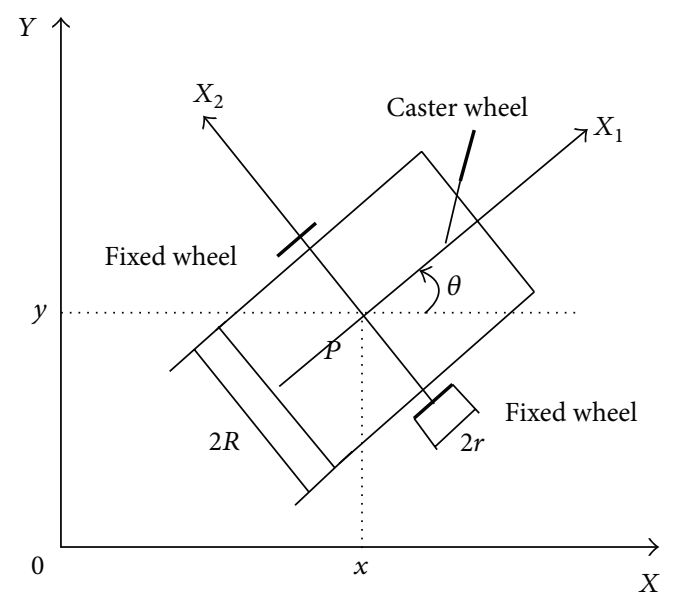

FIGURE 1: Nonholonomic wheeled mobile robot.

velocity, and $\theta$ denotes its heading angle from the horizontal axis. $v$ and $\omega$ are usually regarded as control inputs.

As pointed out in [22], system (1) represents the modeling of the robot in the ideal case. A realistic description of the robot motion in the presence of uncertainties will give rise to far more complex equations requiring involved analysis and synthesis tools. Some results of dealing with such model with uncertain parameters or angle measurement disturbance have been presented; for example, the so-called parking problem of the following system has been addressed with a hybrid feedback control law in [21]

$$
\begin{gathered}
\dot{x}=p_{1}^{*} v \cos \theta, \\
\dot{y}=p_{1}^{*} v \sin \theta, \\
\dot{\theta}=p_{2}^{*} \omega,
\end{gathered}
$$

where $p_{1}^{*}$ and $p_{2}^{*}$ are (unknown) positive parameters determined by the radius of the rear wheels and the distance between them. In addition, Jiang [22] has derived a robust state-feedback controller to drive the states of (2) to the origin with exponential convergence, and in [23], Hong et al. have proposed a finite-time stabilizer for such uncertain system.

As for system (3), considering the control problem of system (1) with angle measurement disturbance (see [22] and references therein),

$$
\begin{gathered}
\dot{x}=v \cos (\theta+\epsilon), \\
\dot{y}=v \sin (\theta+\epsilon), \\
\dot{\theta}=\omega,
\end{gathered}
$$

where $\epsilon$ is a small bias in orientation. As indicated in [20], the second-order approximation of system (3) near the origin is given by

$$
\begin{gathered}
\dot{x}_{\iota}=\left(1-\frac{\epsilon^{2}}{2}\right) v, \\
\dot{y}_{\iota}=\theta_{\iota} v+\epsilon v, \\
\dot{\theta}_{\iota}=\omega,
\end{gathered}
$$


where $\left(x_{t}, y_{t}, \theta_{t}\right)$ represents the state of the above locally approximate model. It was mentioned in [20] that the adaptive control design is applicable to (4) to solve the adaptive regulation of (4) and thus provides a local solution to the parking problem of the robot in the presence of angle measurement errors. And in the literature [22], a switching control strategy has been proposed to globally exponentially regulate all trajectories of system (4) to the equilibrium $(0,0,-\epsilon)$.

But to our knowledge, the stabilization problem of system (3) still remains open up to now. Furthermore, it is the first time to consider the practical stabilization problem for the system containing uncertain parameters and angle measurement disturbance, simultaneously, that is, driving the following system to a prescribed arbitrarily small neighborhood of the equilibrium point $(0,0,0)$ :

$$
\begin{gathered}
\dot{x}=p_{1}^{*} v \cos (\theta+\epsilon), \\
\dot{y}=p_{1}^{*} v \sin (\theta+\epsilon), \\
\dot{\theta}=p_{2}^{*} \omega .
\end{gathered}
$$

As in [22], we can assume that

$$
\begin{aligned}
0<p_{i}^{\min } \leq p_{i}^{*} & \leq p_{i}^{\max }, \quad i=1,2, \\
|\epsilon| & \leq \epsilon^{\max },
\end{aligned}
$$

where $p_{i}^{\min }, p_{i}^{\max }$, and $\epsilon^{\max }$ are all known positive constants.

In this paper, as an example of application, our switching control method to be presented later for the visual feedback model can be easily used to solve the practical stabilization problem of system (5).

2.2. Problem Statement. Generally, for system (1), the state $(x, y)$ can be got from the encoders of motors and other sensors such as ultrasonic sensors ND infrared sensors. However, in the objective world and engineering fields, it is difficult to implement such schemes. Here, the camera shown in Figure 2 is used to measure the position $(x, y)$; its image position is noted as $\left(x_{m}, y_{m}\right)$.

As mentioned in [12-15], we also consider that the movement of the mobile robot (1) can be measured by using a pinhole camera fixed to the ceiling (as shown in Figure 2). Assuming that the camera plane, the image plane, and the robot plane are parallel. There are four coordinate frames, namely, the inertial frame $X-Y-Z$, the camera frame $x-y-z$, the image frame $u-o_{1}-v$, and the attached robot frame $X_{1}-P-X_{2}$. Point $C$ is the crossing point between the optical axis of the camera and $X-Y$ plane. Its coordinate relative to $X-Y$ plane is $\left(c_{x}, c_{y}\right)$. The coordinate of the original point of the camera frame with respect to the image frame is defined by $\left(O_{c_{1}}, O_{c}\right)$. And $(x, y)$ is the coordinate of the mass center $P$ of the robot with respect to $X-Y$ plane.

The pinhole camera model can be expressed as [12]

$$
\left(\begin{array}{l}
x_{m} \\
y_{m}
\end{array}\right)=\left(\begin{array}{cc}
\alpha_{1} & 0 \\
0 & \alpha_{2}
\end{array}\right) R\left(\begin{array}{l}
x-c_{x} \\
y-c_{y}
\end{array}\right)+\left(\begin{array}{l}
O_{c_{1}} \\
O_{c_{2}}
\end{array}\right) \text {, }
$$

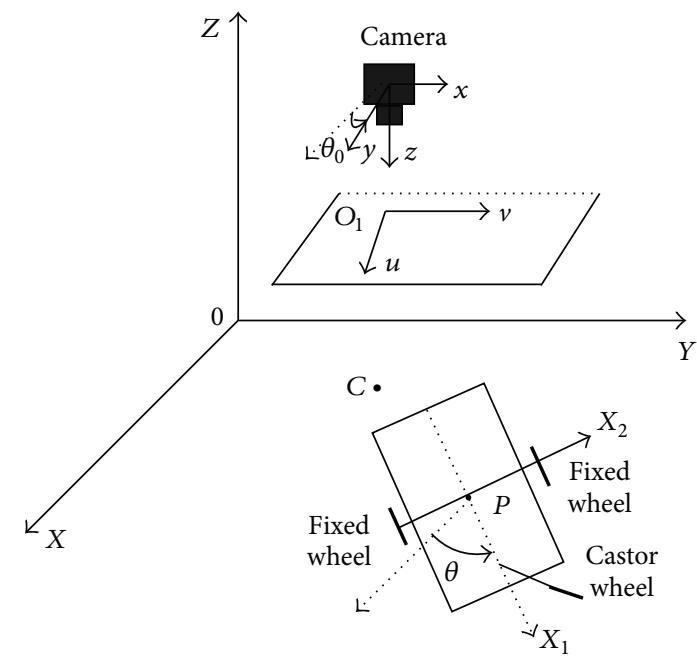

FIgURE 2: Nonholonomic wheeled mobile robot under a fixed camera.

where $\alpha_{1}$ and $\alpha_{2}$ are positive constants, which are dependent on the depth information, focal length, and scalar factors along $u$ axis and $v$ axis, respectively, of the camera system. Here,

$$
R=\left(\begin{array}{cc}
\cos \theta_{0} & \sin \theta_{0} \\
-\sin \theta_{0} & \cos \theta_{0}
\end{array}\right),
$$

where $\theta_{0}$ denotes the angle between $x$-axis and $y$-axis with a positive anticlockwise orientation.

From (1), (7), and (8), by using a simple derivation, the image-based kinematical equation of the robot can be obtained

$$
\begin{gathered}
\dot{x}_{m}=\alpha_{1} v \cos \left(\theta-\theta_{0}\right), \\
\dot{y}_{m}=\alpha_{2} v \sin \left(\theta-\theta_{0}\right), \\
\dot{\theta}=\omega .
\end{gathered}
$$

In the field of visual servoing feedback of robots, usually the camera parameters $\alpha_{1}, \alpha_{2}$ and the angle $\theta_{0}$ can be got by calibration. But this process will take a lot of time, which implies that it is impossible to use this method in high requirement of real time. Therefore, it is necessary to consider how to design a control law in the case of dealing with these uncalibrated parameters.

In this paper, we consider the robust practical stabilization problem of visual servoing feedback model (9) when $\alpha_{1}$, $\alpha_{2}$, and $\theta_{0}$ are all unknown parameters.

Assumption 1. $\alpha_{1}, \alpha_{2}$, and $\theta_{0}$ are bounded, and the bounds of which are known positive constants

$$
\begin{gathered}
0<\alpha_{i}^{\min } \leq \alpha_{i} \leq \alpha_{i}^{\max }, \quad i=1,2, \\
0<\theta_{0}^{\min } \leq \theta_{0} \leq \theta_{0}^{\max }<\frac{\pi}{4} .
\end{gathered}
$$


Remark 2. It is noted that the above assumption is not rigorous because it is not difficult to estimate the bounds of the depth information, focal length, and scalar factors of the camera in advance. As for the angle $\theta_{0}$, seen in Figure 2, we can always assume that it falls within a certain range.

Next, we will introduce the following lemma that is needed for our controller design later.

Lemma 3 (see [25]). For a first-order system

$$
\dot{\eta}=\bar{u}
$$

where $\eta, \bar{u} \in R^{1}$ are state variable and control input, respectively. Taking a continuous, fractional control law

$$
\bar{u}=-k \operatorname{sgn}(\eta)|\eta|^{\beta}
$$

where $k>0, \beta \in(0,1)$ are design parameters. Then the closedloop system $\dot{\eta}=-k \operatorname{sgn}(\eta)|\eta|^{\beta}$ is finite-time stable at the zero equilibrium point.

Proof. See [25].

Remark 4. As illustrated by system (8) in [25], the piecewise control law is continuous everywhere and locally Lipschitz everywhere except for the origin. Therefore, for every initial condition in $\mathbb{R} \backslash\{0\}$, the closed-loop system has a unique solution in forward time.

The control task is to present a switching controller for system (9) such that $\left(x_{m}, y_{m}\right)$ converges to a prescribed arbitrarily small neighborhood of zero, and $\theta(t) \equiv 0$ after a finite time.

\section{Main Results}

In this section, the main results will be presented. To better understand the idea of design, it is useful to first give a careful analysis. Considering system (9) when $\theta(t) \equiv \pi / 4$, it is easy to obtain

$$
\frac{d y_{m}}{d x_{m}}=\frac{\alpha_{2}}{\alpha_{1}} \tan \left(\frac{\pi}{4}-\theta_{0}\right) .
$$

Solving it yields

$$
y_{m}(t)=y_{m}(0)+\frac{\alpha_{2}}{\alpha_{1}}\left(x_{m}(t)-x_{m}(0)\right) \tan \left(\frac{\pi}{4}-\theta_{0}\right),
$$

which implies a line on the $X_{m}-Y_{m}$ plane. Similarly, for the case $\theta(t) \equiv-\pi / 4$, we have

$$
\frac{d y_{m}}{d x_{m}}=-\frac{\alpha_{2}}{\alpha_{1}} \tan \left(\frac{\pi}{4}+\theta_{0}\right) .
$$

Solving it yields

$$
y_{m}(t)=y_{m}(0)-\frac{\alpha_{2}}{\alpha_{1}}\left(x_{m}(t)-x_{m}(0)\right) \tan \left(\frac{\pi}{4}+\theta_{0}\right),
$$

which also implies a line on the $X_{m}-Y_{m}$ plane. By (14) and (16), a good switching controller is expected to drive the robot

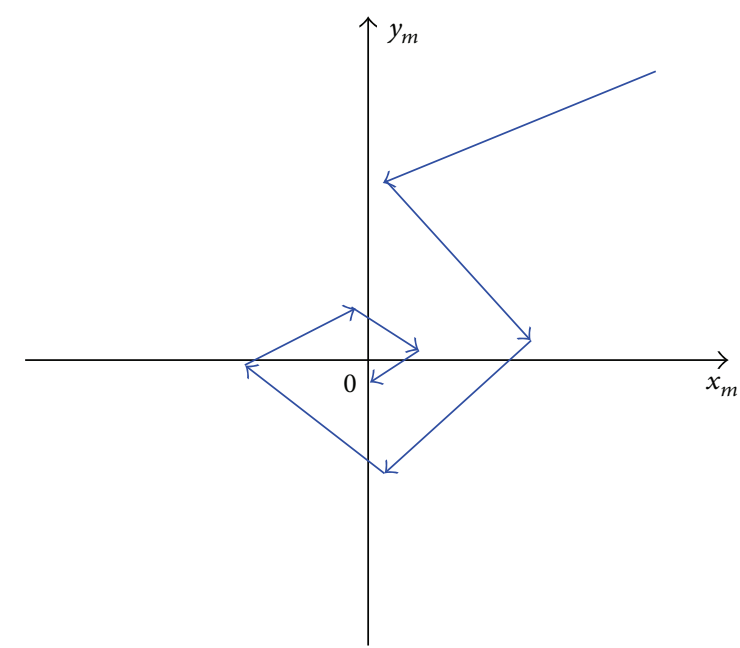

FIGURE 3: The trajectories $\left(x_{m}, y_{m}\right)$ of system (9) under the switching controller.

(9) to an arbitrarily small neighborhood of $(0,0,0)$ according to Figure 3.

The main idea is introduced as follows to show how to design a switching controller for system (9). In every step of the switching process, we always let the angle $\theta(t) \equiv-\pi / 4$ or $\pi / 4$ after some finite time by designing the control input $\omega$ and simultaneously design $v$ to drive $\left|x_{m}(t)\right|\left(\left|y_{m}(t)\right|\right)$ to a small positive constant while $\left|y_{m}(t)\right|\left(\left|x_{m}(t)\right|\right)$ is bounded; their bounds are smaller and smaller. Finally, there must exist a finite time $0<T<+\infty$ such that $\left|x_{m}(T)\right| \leq \varepsilon,\left|y_{m}(T)\right| \leq \varepsilon$ for any arbitrarily small $\varepsilon>0$ given in advance. Then after that, we can let $\left(x_{m}(t), y_{m}(t)\right)$ remain motionless $(v=0)$ and drive $\theta(t)$ to zero in a finite time by designing $\omega$.

The following theorem and its proof will present our switching controller design and stability analysis.

\subsection{Controller Design and Stability Analysis}

Theorem 5. For any given arbitrarily small $\varepsilon>0$ and positive constants $\alpha_{i}^{\mathrm{min}}, \alpha_{i}^{\max }, \theta_{0}^{\min }$, and $\theta_{0}^{\max }$ satisfying Assumption 1, take the following switching controller.

Step $1(0)$. For all $T_{0} \geq 0$, if $\left|x_{m}\left(T_{0}\right)\right| \leq \varepsilon$ and $\left|y_{m}\left(T_{0}\right)\right| \leq \varepsilon$, let

$$
v=0, \quad \omega=-k_{0} \operatorname{sgn}(\theta)|\theta|^{\beta_{0}},
$$

where $k_{0}>0, \beta_{0} \in(0,1)$ are design parameters. If $\theta(t) \equiv 0$ then let $v=\omega=0$ and stop. Otherwise, go to Step $2(2 j-1)$, where $j$ is introduced as a counter, denotes the number of cycles of the switching algorithm beginning with $j=1$. For example, in the first round, $j=1$; in the second round, $j=2$, and so on.

For more clarity, it should be forewarned, in every round of this control algorithm $(j=1,2,3, \ldots)$, the switching process consists of three steps (Step $1(0)$, Step $2(2 j-1)$, Step $3(2 j))$. 
Step $2(2 j-1)$. For all $T_{2 j-1}^{0} \geq 0$, if $\left|x_{m}\left(T_{2 j-1}^{0}\right)\right|>\varepsilon$, let

$$
v=0, \quad \omega=-k_{2 j-1,1} \operatorname{sgn}\left(\theta-\frac{\pi}{4}\right)\left|\theta-\frac{\pi}{4}\right|^{\beta_{2 j-1}},
$$

where $k_{2 j-1,1}>0, \beta_{2 j-1} \in(0,1)$ are design parameters. When $\theta(t) \equiv \pi / 4$ after some finite time, then let

$$
v=-k_{2 j-1,2} x_{m}, \quad \omega=0,
$$

where $k_{2 j-1,2}>0$ is a design parameter. Then, there exists $0 \leq$ $T_{2 j-1}<+\infty$ such that $\left|x_{m}\left(T_{2 j-1}\right)\right| \leq \varepsilon$, and if $\left|y_{m}\left(T_{2 j-1}\right)\right| \leq \varepsilon$, go to Step $1(0)$, else if $\left|y_{m}\left(T_{2 j-1}\right)\right|>\varepsilon$, go to the next step.

Step $3(2 j)$. Let

$$
v=0, \quad \omega=-k_{2 j, 1} \operatorname{sgn}\left(\theta+\frac{\pi}{4}\right)\left|\theta+\frac{\pi}{4}\right|^{\beta_{2 j}},
$$

where $k_{2 j, 1}>0, \beta_{2 j} \in(0,1)$ are design parameters. When $\theta(t) \equiv-\pi / 4$ after some finite time, then let

$$
v=k_{2 j, 2} y_{m}, \quad \omega=0,
$$

where $k_{2 j, 2}>0$ is a design parameter. There exists $0 \leq T_{2 j}<$ $+\infty$ such that $\left|y_{m}\left(T_{2 j}\right)\right| \leq \varepsilon$ as $t \geq T_{2 j}$, and if $\left|x_{m}\left(T_{2 j}\right)\right| \leq \varepsilon$, go to Step $1(0)$, else if $\left|x_{m}\left(T_{2 j}\right)\right|>\varepsilon$, let $j:=j+1$ and go to Step $2(2 j-1)$.

Then, for any initial value $\left(x_{m}(0), y_{m}(0), \theta(0)\right)$, the state $\left(x_{m}(t), y_{m}(t)\right)$ of system (9) can be driven into a prescribed arbitrarily small neighborhood of the origin $D_{\varepsilon} \triangleq\left\{\left(x_{m}, y_{m}\right) \in\right.$ $\left.\mathbb{R}^{2}|| x_{m}|\leq \varepsilon,| y_{m} \mid \leq \varepsilon\right\}$ and stay within it in a finite time; meanwhile, $\theta(t)$ can be stabilized to zero.

Proof. Firstly, for convenience sake, we introduce a positive number $\varepsilon_{0}$ satisfying

$$
\begin{array}{r}
\varepsilon_{0} \leq \varepsilon \cdot \min \left\{\frac{1-1 / \tan ^{2}\left(\theta_{0}^{\min }+(\pi / 4)\right)}{3\left(1+\alpha_{2}^{\max } / \alpha_{1}^{\min }\left(\tan \theta_{0}^{\min }+(\pi / 4)\right)\right)},\right. \\
\left.\frac{1-1 / \tan ^{2}\left(\theta_{0}^{\min }+(\pi / 4)\right)}{3\left(1+\alpha_{1}^{\max } / \alpha_{2}^{\min }\left(\tan \theta_{0}^{\min }+(\pi / 4)\right)\right)}\right\} .
\end{array}
$$

Suppose $\left(x_{m}(0), y_{m}(0)\right) \in D_{\varepsilon}$; it is clear that $\left(x_{m}(t), y_{m}(t)\right)$ will stay within $D_{\varepsilon}$ since $v=0$ in Step 1 (0). According to Lemma 3, there exists a finite time $0<T<+\infty$ such that $\theta(t) \equiv 0$, for all $t \geq T$. Therefore, after $t>T$, the control law $v=\omega=0$ ensures that

$$
\left(x_{m}(t), y_{m}(t)\right) \equiv\left(x_{m}(0), y_{m}(0)\right) \in D_{\varepsilon}, \quad \theta(t) \equiv 0 .
$$

In the case of $\left(x_{m}(0), y_{m}(0)\right) \notin D_{\varepsilon}$, we can use the switching controller which consists of Step $2(2 j-1) \sim$ Step 3 $(2 j)$ again and again, and this control process can be described as follows.

In the first round (i.e., $j=1$ ), if $\left|x_{m}(0)\right|>\varepsilon$, then in Step 2 $(2 j-1)$, from (18) and Lemma 3 , there exists a finite time $T_{1,1}$ such that

$$
\begin{array}{r}
x_{m}\left(T_{1,1}\right)=x_{m}(0), \quad y_{m}\left(T_{1,1}\right)=y_{m}(0), \\
\theta(t) \equiv \frac{\pi}{4}, \quad \forall t \geq T_{1,1},
\end{array}
$$

and for $t \geq T_{1,1}$, from (19), we have

$$
\begin{aligned}
& \dot{x}_{m}=-k_{1,2} \alpha_{1} x_{m} \sin \left(\theta_{0}+\frac{\pi}{4}\right), \\
& \dot{y}_{m}=-k_{1,2} \alpha_{2} x_{m} \cos \left(\theta_{0}+\frac{\pi}{4}\right) .
\end{aligned}
$$

By solving (25) directly, one has

$$
\begin{aligned}
x_{m}(t)= & x_{m}\left(T_{1,1}\right) e^{-k_{1,2} \alpha_{1}\left(t-T_{1,1}\right) \sin \left(\theta_{0}+\pi / 4\right)} \\
= & x_{m}(0) e^{-k_{1,2} \alpha_{1}\left(t-T_{1,1}\right) \sin \left(\theta_{0}+\pi / 4\right)}, \\
y_{m}(t)= & \frac{\alpha_{2}}{\alpha_{1} \tan \left(\theta_{0}+\pi / 4\right)} x_{m}(0) e^{-k_{1,2} \alpha_{1}\left(t-T_{1,1}\right) \sin \left(\theta_{0}+\pi / 4\right)} \\
& +y_{m}(0)-\frac{\alpha_{2}}{\alpha_{1} \tan \left(\theta_{0}+\pi / 4\right)} x_{m}(0) .
\end{aligned}
$$

Let

$$
p \triangleq \frac{\alpha_{2}}{\alpha_{1} \tan \left(\theta_{0}+\pi / 4\right)}, \quad q \triangleq \frac{\alpha_{1}}{\alpha_{2} \tan \left(\theta_{0}+\pi / 4\right)}
$$

Then from Assumption 1, we have

$$
\begin{aligned}
& 0<p \leq \frac{\alpha_{2}^{\max }}{\alpha_{1}^{\min }\left(\tan \theta_{0}^{\min }+\pi / 4\right)} \triangleq p^{\max }, \\
& 0<q \leq \frac{\alpha_{1}^{\max }}{\alpha_{2}^{\min }\left(\tan \theta_{0}^{\min }+\pi / 4\right)} \triangleq q^{\max } .
\end{aligned}
$$

According to (26) and (27), there exists $T_{1,1}<T_{1}<+\infty$ such that

$$
x_{m}\left(T_{1}\right)=\delta_{0}, \quad y_{m}\left(T_{1}\right)=p \delta_{0}+y_{m}(0)-p x_{m}(0),
$$

where $\delta_{0}$ is a given small constant satisfying that $\left|\delta_{0}\right| \leq$ $\min \left\{\varepsilon_{0}, \varepsilon\right\}$. Suppose that $\left|y_{m}\left(T_{1}\right)\right|>\varepsilon$; then go to Step $3(2 j)$.

Similarly, in Step 3 (2j), from (21)-(22) and Lemma 3, we have

$$
\begin{aligned}
y_{m}(t)= & y_{m}\left(T_{2,1}\right) e^{-k_{2,2} \alpha_{2}\left(t-T_{2,1}\right) \sin \left(\theta_{0}+\pi / 4\right)} \\
= & y_{m}\left(T_{1}\right) e^{-k_{2,2} \alpha_{2}\left(t-T_{2,1}\right) \sin \left(\theta_{0}+\pi / 4\right)}, \\
x_{m}(t)= & -\frac{\alpha_{1}}{\alpha_{2} \tan \left(\theta_{0}+\pi / 4\right)} \\
& \times y_{m}\left(T_{1}\right) e^{-k_{2,2} \alpha_{2}\left(t-T_{2,1}\right) \sin \left(\theta_{0}+\pi / 4\right)} \\
& +x_{m}\left(T_{2,1}\right)+\frac{\alpha_{1}}{\alpha_{2} \tan \left(\theta_{0}+\pi / 4\right)} y_{m}\left(T_{1}\right) \\
= & -q y_{m}\left(T_{1}\right) e^{-k_{2,2} \alpha_{2}\left(t-T_{2,1}\right) \sin \left(\theta_{0}+\pi / 4\right)} \\
& +x_{m}\left(T_{1}\right)+q y_{m}\left(T_{1}\right) .
\end{aligned}
$$


According to the above equations, there exists $T_{2,1}<T_{2}<$ $+\infty$ such that

$$
\begin{gathered}
y_{m}\left(T_{2}\right)=\delta_{1}, \\
x_{m}\left(T_{2}\right)=-q \delta_{1}+x_{m}\left(T_{1}\right)+q y_{m}\left(T_{1}\right),
\end{gathered}
$$

where $\delta_{1}$ is a given small constant satisfying that $\left|\delta_{1}\right| \leq\left|\delta_{0}\right|$. Substituting (29) into the above formula, one has

$$
\begin{gathered}
y_{m}\left(T_{2}\right)=\delta_{1}, \\
x_{m}\left(T_{2}\right)=-q \delta_{1}+(1+p q) \delta_{0}+q\left(y_{m}(0)-p x_{m}(0)\right) .
\end{gathered}
$$

If $\left|x_{m}\left(T_{2}\right)\right|>\varepsilon$, let $j=2$, then the closed-loop system continues to go to Step $2(2 j-1)$.

From here, we obtained the general conclusion for $j=$ $2,3,4, \ldots$, there always exist corresponding $0<T_{2 j-1}<+\infty$ and $0<T_{2 j}<+\infty$ such that

$$
\begin{gathered}
x_{m}\left(T_{2 j-1}\right)=\delta_{2 j-2}, \\
y_{m}\left(T_{2 j}\right)=\delta_{2 j-1}, \\
y_{m}\left(T_{2 j-1}\right) \\
=p \delta_{2 j-2}+(1+p q) \\
\quad \times \sum_{k=1}^{j-1}(-1)^{j-1-k}(p q)^{j-1-k}\left(\delta_{2 k-1}-p \delta_{2 k-2}\right) \\
+(-1)^{j-1}(p q)^{j-1}\left(y_{m}(0)-p x_{m}(0)\right), \\
x_{m}\left(T_{2 j}\right) \quad(3 \\
=-q \delta_{2 j-1}+(1+p q) \\
\times\left(\delta_{2 j-2}+\sum_{k=1}^{j-1}(-1)^{j+k} p^{j-1-k} q^{j-k}\left(\delta_{2 k-1}-p \delta_{2 k-2}\right)\right) \\
+(-1)^{j-1} p^{j-1} q^{j}\left(y_{m}(0)-p x_{m}(0)\right),
\end{gathered}
$$

where $\delta_{j}$ is a given small constant and satisfies that $\left|\delta_{j}\right| \leq$ $\left|\delta_{j-1}\right|$, for all $j$. By using (33), we have

$$
\left|x_{m}\left(T_{2 j-1}\right)\right| \leq \varepsilon, \quad\left|y_{m}\left(T_{2 j}\right)\right| \leq \varepsilon, \quad \forall j .
$$

On the other hand, from Assumption 1, (27), and (28), one has

$$
\begin{aligned}
0 & <\frac{1}{\tan ^{2}\left(\theta_{0}^{\max }+\pi / 4\right)} \\
& \leq p q=\frac{1}{\tan ^{2}\left(\theta_{0}+\pi / 4\right)} \\
& \leq \frac{1}{\tan ^{2}\left(\theta_{0}^{\min }+\pi / 4\right)}<1 .
\end{aligned}
$$

Then from (34), one can obtain

$$
\begin{aligned}
& \left|y_{m}\left(T_{2 j-1}\right)\right| \\
& \leq p \varepsilon_{0}+(1+p q)(1+p) \varepsilon_{0} \\
& \quad \times \sum_{k=1}^{j-1}(p q)^{j-1-k}+(p q)^{j-1}\left|y_{m}(0)-p x_{m}(0)\right| \\
& \leq p \varepsilon_{0}+\frac{(1+p q)(1+p) \varepsilon_{0}}{1-p q} \\
& \quad+(p q)^{j-1}\left|y_{m}(0)-p x_{m}(0)\right| .
\end{aligned}
$$

Because $\left|y_{m}(0)-p x_{m}(0)\right|$ is bounded and $0<p q<1$, there must exist $J_{1}<+\infty$ such that

$$
\begin{aligned}
\left|y_{m}\left(T_{2 j-1}\right)\right| & \leq p \varepsilon_{0}+\frac{2(1+p) \varepsilon_{0}}{1-p q}+\varepsilon_{0} \\
& =\frac{(1+p)(1-p q+2) \varepsilon_{0}}{1-p q} \\
& \leq \frac{3(1+p) \varepsilon_{0}}{1-p q}, \quad j \geq J_{1} .
\end{aligned}
$$

By using (22), (27), (28), and (37), one has

$$
\varepsilon_{0} \leq \varepsilon \cdot \min \left\{\frac{1-p q}{3(1+p)}, \frac{1-p q}{3(1+q)}\right\} .
$$

Hence,

$$
\left|y_{m}\left(T_{2 j-1}\right)\right| \leq \varepsilon, \quad j \geq J_{1} .
$$

Similarly, from (35), we have

$$
\begin{aligned}
\left|x_{m}\left(T_{2 j}\right)\right| \leq & q \varepsilon_{0}+(1+p q) \\
& \times\left(\varepsilon_{0}+(1+p) \varepsilon_{0} \sum_{k=1}^{j-1} p^{j-1-k} q^{j-k}\right) \\
& +p^{j-1} q^{j}\left|y_{m}(0)-p x_{m}(0)\right| \\
\leq & q \varepsilon_{0}+(1+p q)\left(1+\frac{(1+p) q}{1-p q}\right) \varepsilon_{0} \\
& +q(p q)^{j-1}\left|y_{m}(0)-p x_{m}(0)\right| .
\end{aligned}
$$

As the same with (41), there must exist $J_{2}<+\infty$ such that

$$
\begin{aligned}
\left|x_{m}\left(T_{2 j}\right)\right| & \leq q \varepsilon_{0}+2\left(1+\frac{(1+p) q}{1-p q}\right) \varepsilon_{0}+\varepsilon_{0} \\
& \leq \frac{(1+q)(1-p q+2) \varepsilon_{0}}{1-p q} \\
& \leq \frac{3(1+q) \varepsilon_{0}}{1-p q}, \quad j \geq J_{2} .
\end{aligned}
$$


Applying (40) again, we have

$$
\left|x_{m}\left(T_{2 j}\right)\right| \leq \varepsilon, \quad j \geq J_{2} .
$$

Therefore, combining (36), (41), and (44), it is obvious that there must exist $J=\max \left\{J_{1}, J_{2}\right\}<+\infty$ and a finite time $T_{J}$ such that

$$
\left|x_{m}\left(T_{J}\right)\right| \leq \varepsilon, \quad\left|y_{m}\left(T_{J}\right)\right| \leq \varepsilon, \quad j \geq J
$$

which means that $\left(x_{m}(t), y_{m}(t)\right)$ can be driven into the region $D_{\varepsilon}$ sooner or later; then go to Step $1(0)$. And this completes the proof of Theorem 5 .

3.2. An Example of Application. In this subsection, we will design a controller for system (5) by applying the switching control method proposed in Theorem 5.

Theorem 6. For any given arbitrarily small $\varepsilon_{00}>0$ and positive constants $p_{i}^{\min }, p_{i}^{\max }$, and $\epsilon^{\max }$ satisfying (6), suppose

$$
\epsilon^{\max }<\varphi<\frac{\pi}{4}-\epsilon^{\max }
$$

takes the following switching controller.

Step 1(00). For all $T_{00} \geq 0$, if $\left|x\left(T_{00}\right)\right| \leq \varepsilon_{00}$ and $\left|y\left(T_{00}\right)\right| \leq \varepsilon_{00}$, let

$$
v=0, \quad \omega=-l_{0} \operatorname{sgn}(\theta)|\theta|^{\gamma_{0}},
$$

where $l_{0}>0, \gamma_{0} \in(0,1)$ are design parameters. If $\theta(t) \equiv 0$, then let $v=\omega=0$ and stop. Otherwise, go to Step $2(2 n-1)$.

Step $2(2 n-1)$. If $\left|x\left(T_{2 n-1}^{0}\right)\right|>\varepsilon_{00}$, for all $T_{2 n-1}^{0} \geq 0$, let

$$
\begin{gathered}
\nu=0, \\
\omega=-l_{2 n-1,1} \operatorname{sgn}\left(\theta+\varphi-\frac{\pi}{4}\right)\left|\theta+\varphi-\frac{\pi}{4}\right|^{\gamma_{2 n-1}},
\end{gathered}
$$

where $l_{2 n-1,1}>0, \gamma_{2 n-1} \in(0,1)$ are design parameters. When $\theta(t)+\varphi \equiv \pi / 4$ after some finite time, then let

$$
v=-l_{2 n-1,2} x, \quad \omega=0,
$$

where $l_{2 n-1,2}>0$ is a design parameter. Then, there exists $0 \leq$ $T_{2 n-1}<+\infty$ such that $\left|x\left(T_{2 n-1}\right)\right| \leq \varepsilon_{00}$, and if $\left|y\left(T_{2 n-1}\right)\right| \leq \varepsilon_{00}$, go to Step $1(00)$, else if $\left|y\left(T_{2 n-1}\right)\right|>\varepsilon_{00}$, go to Step $3(2 n)$.

Step $3(2 n)$. Let

$$
\begin{gathered}
v=0 \\
\omega=-l_{2 n, 1} \operatorname{sgn}\left(\theta+\varphi+\frac{\pi}{4}\right)\left|\theta+\varphi+\frac{\pi}{4}\right|^{\gamma_{2 n}},
\end{gathered}
$$

where $l_{2 n, 1}>0, \gamma_{2 n} \in(0,1)$ are design parameters. When $\theta(t)+\varphi \equiv-\pi / 4$ after some finite time, then let

$$
v=l_{2 n, 2} y, \quad \omega=0,
$$

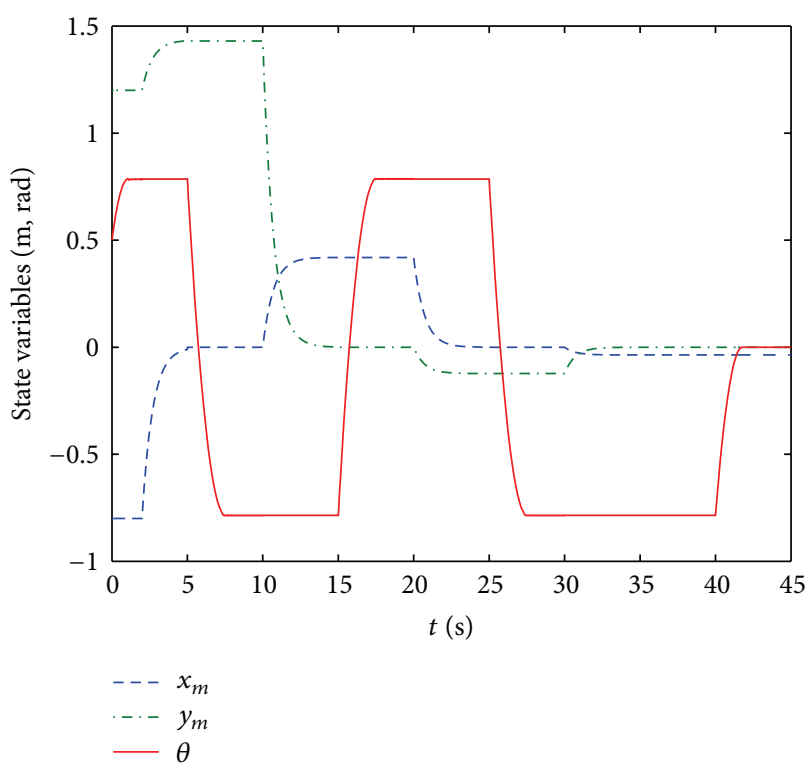

FIGURE 4: State variables.

where $l_{2 n, 2}>0$ is a design parameter. There exists $0 \leq T_{2 n}<$ $+\infty$ such that $\left|y\left(T_{2 n}\right)\right| \leq \varepsilon_{00}$, and if $\left|x\left(T_{2 n}\right)\right| \leq \varepsilon_{00}$, go to Step 1 (00), else if $\left|x\left(T_{2 n}\right)\right|>\varepsilon_{00}$, let $n:=n+1$ and go to Step 2 $(2 n-1)$.

Then, for any initial value $(x(0), y(0), \theta(0))$, the state $(x(t), y(t))$ of system (5) can be driven into a prescribed arbitrarily small neighborhood of the origin $D_{\varepsilon_{00}} \triangleq\{(x(t), y(t)) \epsilon$ $\left.\mathbb{R}^{2}|| x(t)\left|\leq \varepsilon_{00},\right| y(t) \mid \leq \varepsilon_{00}\right\}$ and stay within it; meanwhile, $\theta(t)$ can be stabilized to zero in a finite time.

Remark 7. The proof of Theorem 6 is similar to that of Theorem 5 and thus is omitted. In fact, system (5) can be rewritten as

$$
\begin{gathered}
\dot{x}=p_{1}^{*} v \cos (\theta+\varphi-(\varphi-\epsilon)), \\
\dot{y}=p_{1}^{*} v \sin (\theta+\varphi-(\varphi-\epsilon)), \\
\dot{\theta}=p_{2}^{*} \omega .
\end{gathered}
$$

Since $\epsilon$ denotes a small bias in orientation, it is not rigorous to assume that $|\epsilon|$ has a small upper bound less than $\pi / 8$, that is, $\epsilon^{\max } \leq \pi / 8$. Then from (6) and (46), one has

$$
0<\varphi-\epsilon^{\max } \leq \varphi-\epsilon \leq \varphi+\epsilon^{\max }<\frac{\pi}{4} .
$$

Let

$$
\alpha_{1}=\alpha_{2}=p_{1}^{*}, \quad \theta_{0}=\varphi-\epsilon,
$$

and note that it is also valid to use finite-time control technique (Lemma 3) for $\theta$-subsystem in spite of the uncertain parameter $p_{2}^{*}$. Hence, system (52) can be seen as a special case of our visual servoing model (9).

\section{Simulations}

In this section, the switching controller proposed in Theorem 5 is used to show how to stabilize the state 


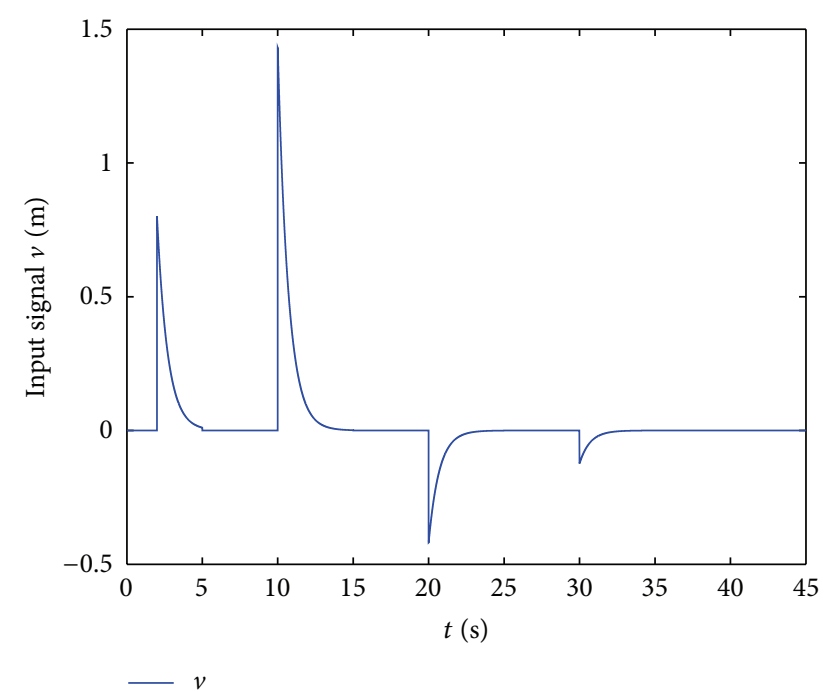

FIGURE 5: Control input $v$.

$\left(x_{m}(t), y_{m}(t), \theta(t)\right)$ of the uncertain visual feedback system (9) into a prescribed arbitrarily small neighborhood of the zero equilibrium point. We will demonstrate the effectiveness of our methods by a numerical example.

In the following simulation, we assume that $\varepsilon=0.05$, $\varepsilon_{0}=0.04, \delta_{j}=0.02$, (for all $j \in\{1,2,3, \ldots\}$ ), $\alpha_{i}^{\min }=1$, $\alpha_{i}^{\max }=2,(i=1,2), \theta_{0}^{\min }=0.2, \theta_{0}^{\max }=0.6, \alpha_{1}=\alpha_{2}=1.5$, and $\theta_{0}=0.5$. According to Theorem 5 , we can choose design parameters $k_{0}=k_{2 j-1,1}=k_{2 j-1,2}=k_{2 j, 1}=k_{2 j, 2}=1, \beta_{0}=$ $\beta_{2 j-1}=\beta_{2 j}=0.5$, for all $j$. The initial condition of system (9) is $\left(x_{m}(0), y_{m}(0), \theta(0)\right)=(-0.8,1.2,0.5)$.

Figures 4-7 show some simulation results with MATLAB. Figure 4 shows that the state variable $\left(x_{m}, y_{m}\right)$ can be driven into the region $D_{\varepsilon}$ step by step by using the switching controller Step 1(0) Step $3(2 j)$ in Theorem 5. When $t \geq 33 \mathrm{~s}$, one has $\left|x_{m}(t)\right| \leq \varepsilon$ and $\left|y_{m}(t)\right| \leq \varepsilon$. While $\theta(t)$ is always switched between $\pi / 4$ and $-\pi / 4$, and finally, $\theta(t) \equiv 0$ as $t \geq 40 \mathrm{~s}$.

Figures 5-6 show the responses of the switching control inputs $v$ and $\omega$ as time passes. And as shown in Figure 7, by a three-dimensional simulation image, we can more clearly see how the position state $\left(x_{m}(t), y_{m}(t)\right)$ enters into the prescribed arbitrarily small neighborhood of the zero $D_{\varepsilon}$ step by step.

\section{Conclusion}

In this paper, a switching controller is presented for the practical stabilization problem of a class of nonholonomic mobile robots based on visual servoing model with uncalibrated camera parameters. The best innovation of this paper is that the controller design is based on the less strict hypothesis (assuming that all the camera parameters are unknown) compared with the previous results [12-15], which is more practical in real world implementations. In addition, the new design method is directly based on the original system model

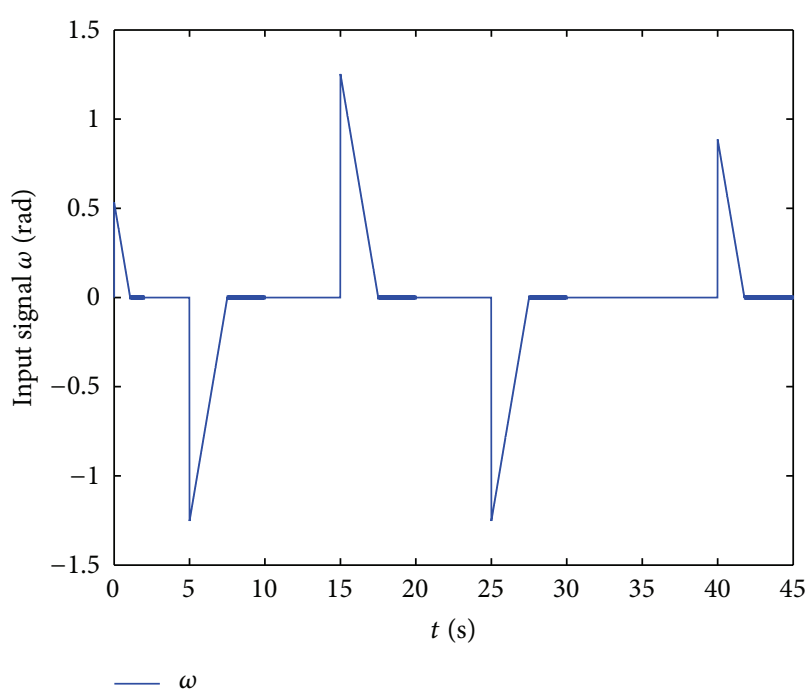

FIgURE 6: Control input $\omega$.

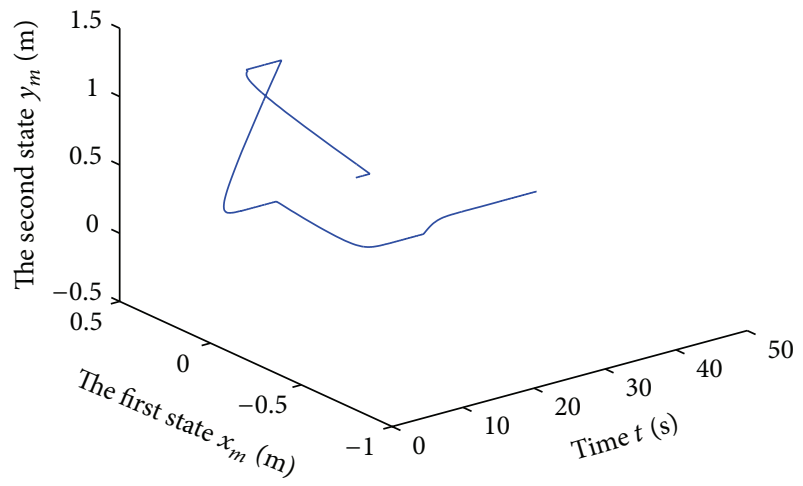

Figure 7: Position state $\left(x_{m}, y_{m}\right)$.

without any local change of coordinates or feedback; it is valid to avoid appearance of singularity.

\section{Acknowledgments}

This paper was supported by the Science and Technology Support Program of Jiangsu Province (BE2012055), the Postdoctoral Fund of Hohai University (2016-412057), the Youth Fund of Hohai University (2009B32314), the Changzhou Science and Technology Support (Industry) Project (CE20120080, CE20120073), the Open Projects of Changzhou Key Laboratory of Sensor Networks and Environmental Sensing (CZSN201207), and the China Postdoctoral Science Foundation (2013M531263). The simple result of this paper was presented in the 31st Chinese Control Conference, Hefei, China, 2012. 


\section{References}

[1] R. W. Brockett, "Asymptotic stability and feedback stabilization," in Differential Geometric Control Theory, R. W. Brockett, R. S. Millman, and H. J. Sussmann, Eds., vol. 27, pp. 181-208, Birkhäuser, Boston, Mass, USA, 1983.

[2] R. M. Murray and S. S. Sastry, "Nonholonomic motion planning: steering using sinusoids," IEEE Transactions on Automatic Control, vol. 38, no. 5, pp. 700-716, 1993.

[3] Y. P. Tian and S. Li, "Exponential stabilization of nonholonomic dynamic systems by smooth time-varying control," Automatica, vol. 38, no. 8, pp. 1139-1146, 2002.

[4] A. Teel, R. Murry, and G. Walsh, "Nonholonomic control systems: from steering to stabilization with sinusoids," in Proceedings of the 31st IEEE Conference on Decision and Control, vol. 2, pp. 1603-1609, 1992.

[5] O. J. Sørdalen and O. Egeland, "Exponential stabilization of nonholonomic chained systems," IEEE Transactions on Automatic Control, vol. 40, no. 1, pp. 35-49, 1995.

[6] J. P. Hespanha, "Stabilization of the nonholonomic integrator via logic-based switching," Tech. Rep., Yale University, 1995.

[7] A. P. Aguiar and A. Pascoal, "Stabilization of the extended nonholonomic double integrator via logic based hybridcontrol," in Proceedings of 6th International IFAC Symposium on Robot Control (SYROCO '00), Vienna, Austria, September 2000.

[8] A. Astolfi, "Discontinuous control of nonholonomic systems," Systems \& Control Letters, vol. 27, no. 1, pp. 37-45, 1996.

[9] A. M. Bloch and S. Drakunov, "Stabilization of a nonholonomic system via sliding modes," in Proceedings of the 33rd IEEE Conference on Decision and Control, vol. 3, pp. 2961-2963, December 1994.

[10] C. Canudas de Wit and O. J. Sørdalen, "Exponential stabilization of mobile robots with nonholonomic constraints," IEEE Transactions on Automatic Control, vol. 37, no. 11, pp. 1791-1797, 1992.

[11] M. C. Laiou and A. Astolfi, "Discontinuous control of highorder generalized chained systems," Systems \& Control Letters, vol. 37, no. 5, pp. 309-322, 1999.

[12] Z. Liang and C. Wang, "Robust stabilization of nonholonomic chained form systems with uncertainties," Acta Automatica Sinica, vol. 37, no. 2, pp. 129-142, 2011.

[13] F. Yang and C. Wang, "Adaptive stabilization for uncertain nonholonomic dynamic mobile robots based on visual servoing feedback," Acta Automatica Sinica, vol. 37, no. 7, pp. 857-864, 2011.

[14] C. Wang, Z. Liang, and Y. Liu, "Dynamic feedback robust regulation of nonholonomic mobile robots based on visual servoing," in Proceedings of the 48th IEEE Conference on Decision and Control held jointly with 28th Chinese Control Conference (CDC/CCC '09), pp. 4384-4389, December 2009.

[15] C. Wang, Y. Mei, Z. Liang, and Q. Jia, "Dynamic feedback tracking control of non-holonomic mobile robots with unknown camera parameters," Transactions of the Institute of Measurement and Control, vol. 32, no. 2, pp. 155-169, 2010.

[16] B. D’Andrea-Novel, G. Bastin, and G. Campion, "Dynamic feedback linearization of nonholonomic wheeled mobile robots," in Proceedings of IEEE International Conference on Robotics and Automation, pp. 2527-2532, May 1992.

[17] M. Fliess, J. L. Lévine, P. Martin, and P. Rouchon, "Flatness and defect of non-linear systems: introductory theory and examples," International Journal of Control, vol. 61, no. 6, pp. 1327-1361, 1995.
[18] T. Liu and Z.-P. Jiang, "Distributed formation control of nonholonomic mobile robots without global position measurements," Automatica, vol. 49, no. 2, pp. 592-600, 2013.

[19] Z. D. Sun, S. S. Ge, W. Huo, and T. H. Lee, "Stabilization of nonholonomic chained systems via nonregular feedback linearization," Systems \& Control Letters, vol. 44, no. 4, pp. 279289, 2001.

[20] P. Morin, J. Pomet, and C. Samson, "Developments in time-varying feedback stabilization of nonlinear systems," in Preprints of Nonlinear Control Systems Design Symposium (NOLCOS '98), pp. 587-594, Enschede, The Netherlands, 1998.

[21] J. P. Hespanha, D. Liberzon, and A. S. Morse, "Logic-based switching control of a nonholonomic system with parametric modeling uncertainty," Systems \& Control Letters, vol. 38, no. 3, pp. 167-177, 1999.

[22] Z. P. Jiang, "Robust exponential regulation of nonholonomic systems with uncertainties," Automatica, vol. 36, no. 2, pp. 189209, 2000.

[23] Y. Hong, J. Wang, and Z. Xi, "Stabilization of uncertain chained form systems within finite settling time," IEEE Transactions on Automatic Control, vol. 50, no. 9, pp. 1379-1384, 2005.

[24] G. Campion, G. Bastin, and B. D’Andréa-Novel, "Structural properties and classification of kinematic and dynamic models of wheeled mobile robots," IEEE Transactions on Robotics and Automation, vol. 12, no. 1, pp. 47-62, 1996.

[25] S. P. Bhat and D. S. Bernstein, "Continuous finite-time stabilization of the translational and rotational double integrators," IEEE Transactions on Automatic Control, vol. 43, no. 5, pp. 678-682, 1998. 


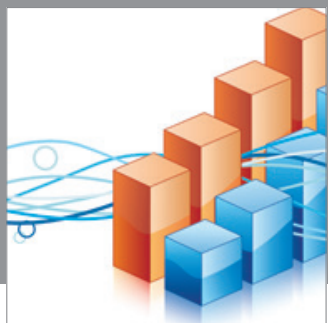

Advances in

Operations Research

mansans

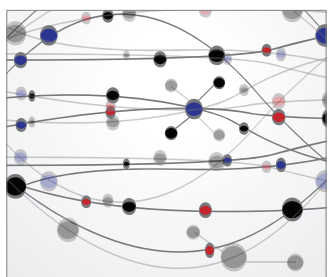

The Scientific World Journal
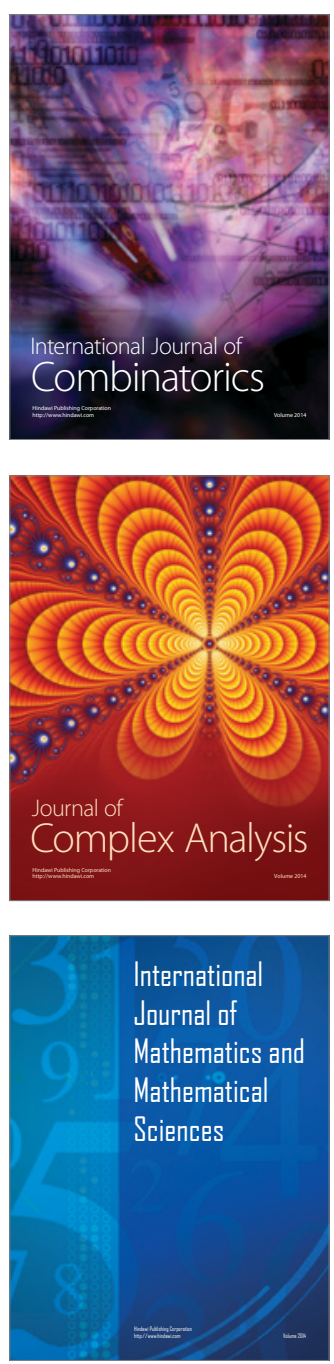
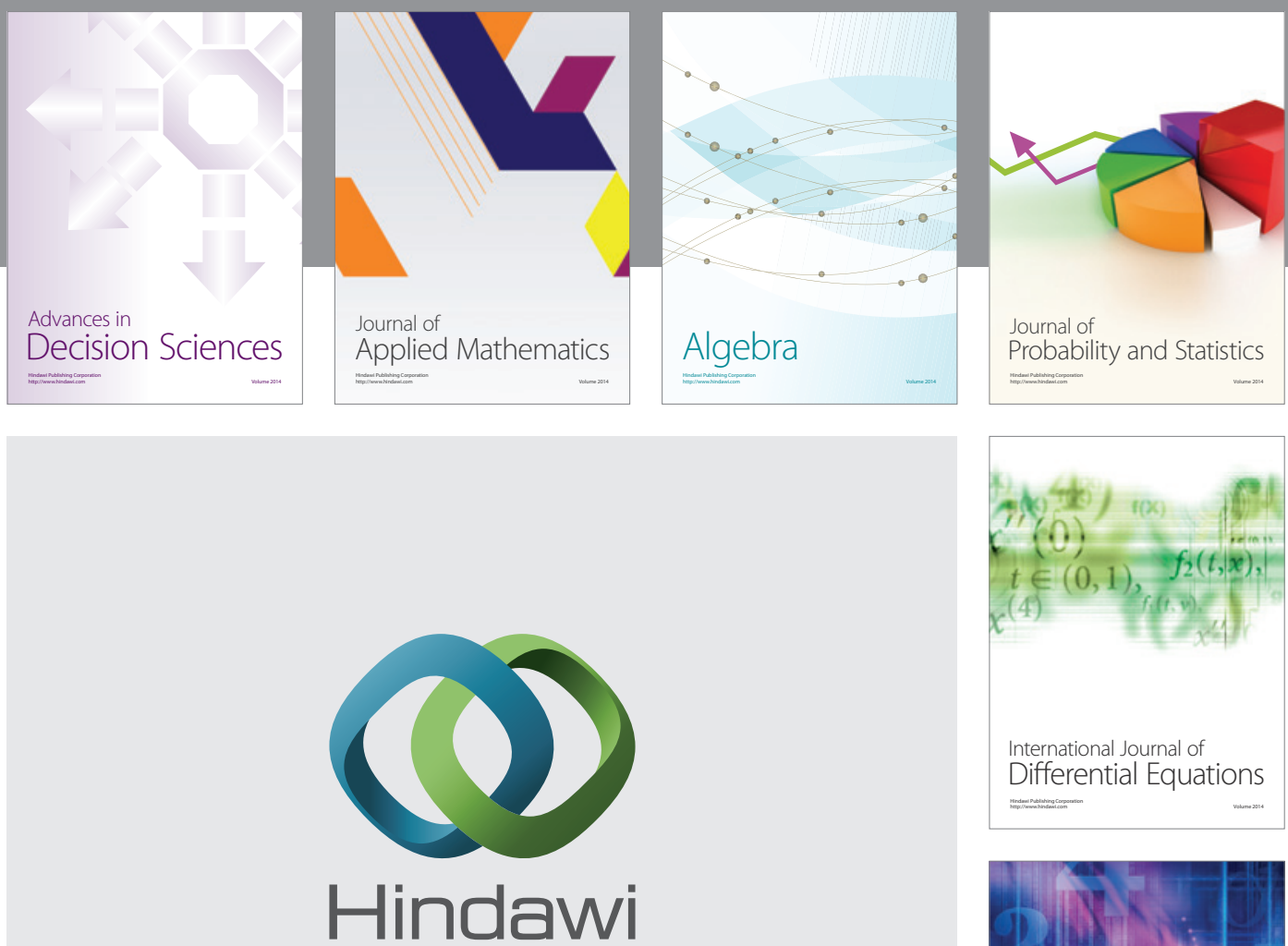

Submit your manuscripts at http://www.hindawi.com
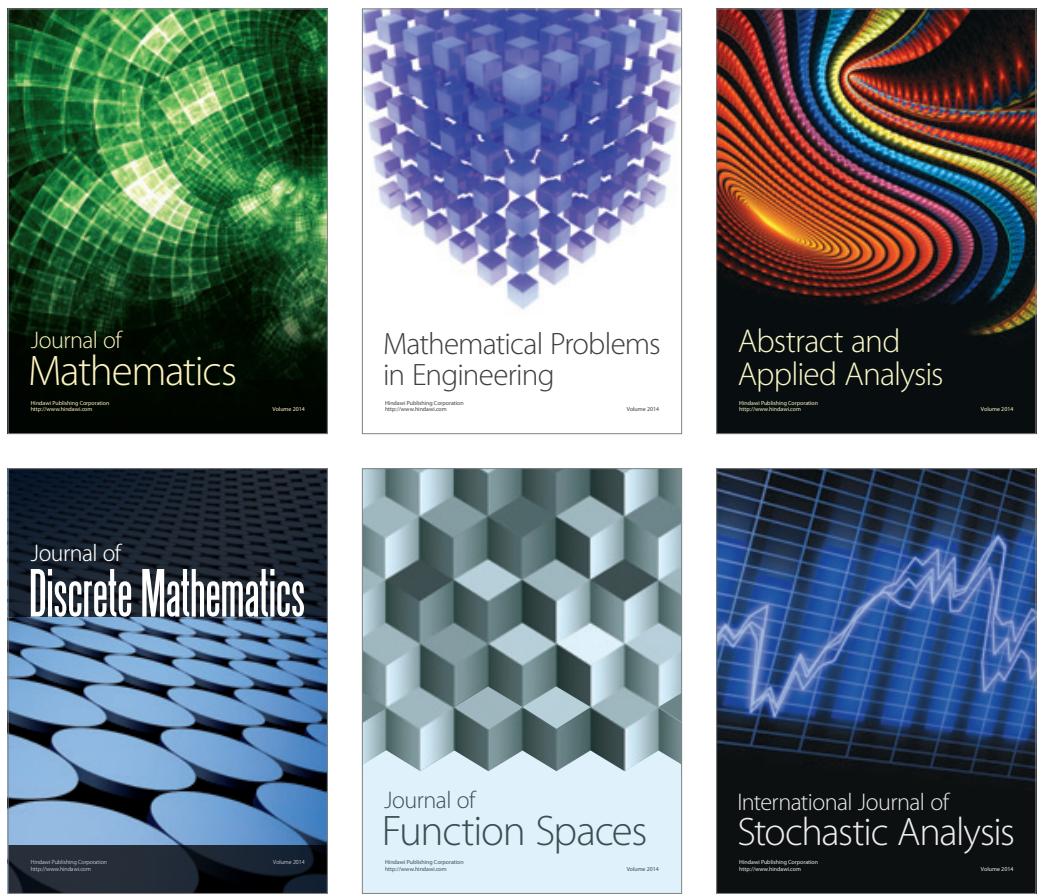

Journal of

Function Spaces

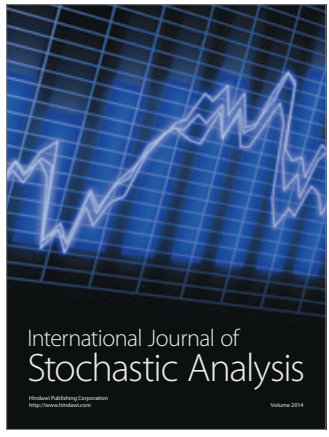

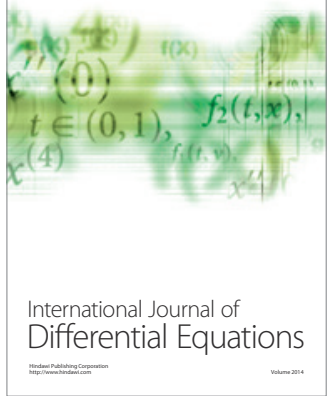
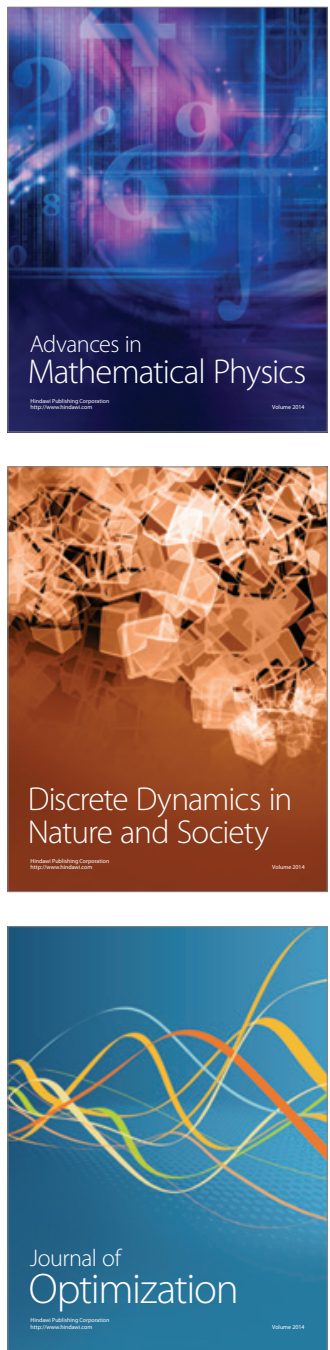